\section{One and a half million hematopoietic stem cell transplants: continuous and differential improvement in worldwide access with the use of non-identical family donors}

\author{
Dietger Niederwieser, ${ }^{1,2,3}$ Helen Baldomero, ${ }^{4}$ Nosa Bazuaye, ${ }^{5,6}$ \\ Caitrin Bupp, ${ }^{7}$ Naeem Chaudhri, ${ }^{8,9}$ Selim Corbacioglu, ${ }^{10,11}$ Alaa Elhaddad,,12 \\ Cristóbal Frutos, ${ }^{13,14}$ Sebastian Galeano, ${ }^{13,15}$ Nada Hamad, ${ }^{16,17}$ Amir Ali \\ Hamidieh, ${ }^{8,18}$ Shahrukh Hashmi, ${ }^{19,20}$ Aloysius Ho, ${ }^{21,22}$ Mary M. Horowitz, ${ }^{23}$ \\ Minako lida, ${ }^{21,24}$ Gregorio Jaimovich, ${ }^{13,25}$ Amado Karduss, ${ }^{13,26}$ \\ Yoshihisa Kodera, ${ }^{21,24}$ Nicolaus Kröger, ${ }^{10,27}$ Regis Peffault de Latour, ${ }^{10,28}$ \\ Jong Wook Lee, ${ }^{21,29}$ Juliana Martínez-Rolón, ${ }^{13,30}$ Marcelo C. Pasquini, ${ }^{23}$ \\ Jakob Passweg, ${ }^{10,31}$ Kristjan Paulson, ${ }^{32}$ Adriana Seber, ${ }^{13,33}$ John A. \\ Snowden, ${ }^{10,34}$ Alok Srivastava, ${ }^{21,35}$ Jeff Szer, ${ }^{16,36}$ Daniel Weisdorf, ${ }^{7,37}$ \\ Nina Worel, ${ }^{38}$ Mickey B. C. Koh, ${ }^{39,40}$ Mahmoud Aljurf, ${ }^{8,41}$ Hildegard Greinix, ${ }^{42}$ \\ Yoshiko Atsuta ${ }^{21,43}$ and Wael Saber ${ }^{23}$ for the Worldwide Network of Blood and \\ Marrow Transplantation WBMT
}

${ }^{1}$ University of Leipzig, Germany; ${ }^{2}$ Aichi Medical University School of Medicine, Nagakute, Japan; ${ }^{3}$ Lithuanian University of Health Sciences, Kaunas, Lithuania; ${ }^{4}$ The Worldwide Network of Blood and Marrow Transplantation (WBMT) Transplant Activity Survey Office, University Hospital, Basel, Switzerland; ${ }^{5}$ African Blood and Marrow Transplantation Group - AfBMT, Bern, Switzerland; ${ }^{6}$ University of Benin Teaching Hospital, Benin, Nigeria; ${ }^{7}$ CIBMTR (Center for International Blood and Marrow Transplant Research), National Marrow Donor Program/Be The Match, Minneapolis, MN; USA; ${ }^{8}$ The Eastern Mediterranean Blood and Marrow Transplant Group (EMBMT), King Faisal Specialist Hospital \& Research Center, Riyadh, Saudi Arabia; ${ }^{9}$ Oncology Center King Faisal Specialist Hospital \& Research Center, Riyadh, Saudi Arabia; ${ }^{10}$ European Society for Blood and Marrow Transplantation (EBMT), Barcelona, Spain; ${ }^{11}$ Department of Pediatric Hematology, Oncology and Stem Cell Transplantation, University of Regensburg, Regensburg, Germany; ${ }^{12}$ Department of Pediatric Oncology and Stem Cell Transplantation Unit, Cairo University, Cairo, Egypt; ${ }^{13}$ Latin American Blood and Marrow Transplantation Group - LABMT, Bern, Switzerland; ${ }^{14}$ Cristóbal Frutos, Instituto de Previsión Social, Asunción, Paraguay; ${ }^{15}$ Sebastian Galeano, Hospital Británico, Montevideo, Uruguay; ${ }^{16}$ Australasian Bone Marrow Transplant Recipient Registry (ABMTRR), St. Vincent's Hospital Sydney, Sydney, New South Wales, Australia; ${ }^{17}$ St. Vincent's Health Network, Kinghorn Cancer Center, Sydney, New South Wales, Australia; ${ }^{18}$ Pediatric Cell Therapy Research Center, Tehran University of Medical Sciences, Tehran, Iran; ${ }^{19}$ Sheikh Shakhbout Medical City, Abu Dhabi, UAE; ${ }^{20}$ MAYO Clinic, Rochester, MN, USA; ${ }^{21}$ The Asia Pacific Blood and Marrow Transplant Group (APBMT), Aichi Medical University School of Medicine, Nagakute, Japan; ${ }^{22}$ Singapore General Hospital Singapore, Singapore; ${ }^{23}$ CIBMTR, Medical College of Wisconsin, Milwaukee, WI, USA; ${ }^{24}$ Aichi Medical University School of Medicine, Deptartment of Promotion for Blood and Marrow Plantation, Nagakute, Japan; ${ }^{25}$ Fundación Favaloro, Sanatorio Anchorena, ITAC, Buenos Aires, Argentina; ${ }^{26}$ Instituto de Cancerología-Clínica Las Américas, Medellín, Colombia; ${ }^{27}$ Department of Stem Cell Transplantation, University Medical Center Hamburg, Hamburg, Germany; ${ }^{28}$ Saint-Louis Hospital, 1 avenue Claude Vellefaux, Paris, France; ${ }^{29}$ Seoul St. Mary's Hospital, The Catholic University of Korea, Seoul, Republic of Korea; ${ }^{30}$ FUNDALEU, Buenos Aires, Argentina; ${ }^{31}$ Klinik für Hämatologie, Universitätsspital Basel, Basel, Switzerland; ${ }^{32}$ Cancercare Manitoba and the University of Manitoba and Cell Therapy Transplant Canada (CTTC), Winnipeg, Manitoba, Canada; ${ }^{33}$ Pediatric Department, Hospital Samaritano, Sao Paulo, Brazil; ${ }^{34}$ Department of Hematology, Sheffield Teaching Hospitals NHS Foundation Trust, Sheffield, UK; ${ }^{35}$ Christian Medical College, Vellore, India; ${ }^{36}$ Peter MacCallum Cancer Center and Royal Melbourne Hospital, Parkville, Vicoria, Australia; ${ }^{37}$ University of Minnesota, Minneapolis, MN, USA; ${ }^{38}$ Medical University of Vienna, Department of Blood Group Serology and Transfusion Medicine, Vienna, Austria; ${ }^{39}$ Infection and Immunity Clinical Academic Group St George's Hospital and Medical School, London, UK; ${ }^{40}$ Academic Cell Therapy Facility and Programme Health Sciences Authority Singapore, Singapore; ${ }^{41} \mathrm{King}$ Faisal Specialist Hospital and Research Center, Riyadh, Saudi Arabia; ${ }^{42}$ Medical University of Graz, Division of Hematology, Graz, Austria and ${ }^{43}$ Japanese Data Center for Hematopoietic Cell Transplantation (JDCHCT),

Nagoya, Japan
Ferrata Storti Foundation

Haematologica 2022

Volume 107(5):1045-1053

\section{Correspondence:}

DIETGER NIEDERWIESER

dietger.niederwieser@medizin.uni-leipzig.de

Received: May 13, 2021.

Accepted: July 21, 2021.

Pre-published: August 12, 2021.

https://doi.org/10.3324/haematol.2021.279189

(C)2022 Ferrata Storti Foundation

Material published in Haematologica is covered by copyright. All rights are reserved to the Ferrata Storti Foundation. Use of published material is allowed under the following terms and conditions:

https://creativecommons.org/licenses/by-nc/4.0/legalcode. Copies of published material are allowed for personal or internal use. Sharing published material for non-commercial purposes is subject to the following conditions:

https://creativecommons.org/licenses/by-nc/4.0/legalcode, sect. 3. Reproducing and sharing published material for commercial purposes is not allowed without permission in writing from the publisher. 
he Worldwide Network of Blood and Marrow Transplantation (WBMT) pursues the mission of promoting hematopoietic cell transplantation (HCT) for instance by evaluating activities through member societies, national registries and individual centers. In 2016, 82,718 first HCT were reported by 1,662 HCT teams in 86 of the 195 World Health Organization member states representing a global increase of $6.2 \%$ in autologous HCT and $7.0 \%$ in allogeneic HCT and bringing the total to 1,298,897 procedures. Assuming a frequency of 84,000/year, 1.5 million HCT were performed by 2019 since 1957. Slightly more autologous (53.5\%) than allogeneic and more related (53.6\%) than unrelated HCT were reported. A remarkable increase was noted in haploidentical related HCT for leukemias and lymphoproliferative diseases, but even more in non-malignant diseases. Transplant rates (TR; HCT/10 million population) varied according to region reaching 560.8 in North America, 438.5 in Europe, 76.7 in Latin America, 53.6 in South East Asia/Western Pacific (SEA/WPR) and 27.8 in African/East Mediterranean (AFR/EMR). Interestingly, haploidentical TR amounted to $32 \%$ in SEA/WPR and $26 \%$ in Latin America, but only $14 \%$ in Europe and EMR and $4.9 \%$ in North America of all allogeneic HCT. HCT team density (teams/10 million population) was highest in Europe (7.7) followed by North America (6.0), SEA/WPR (1.9), Latin America (1.6) and AFR/EMR (0.4). HCT are increasing steadily worldwide with narrowing gaps between regions and greater increase in allogeneic compared to autologous activity. While related HCT is rising, largely due to increase in haploidentical HCT, unrelated HCT is plateauing and cord blood HCT is in decline.

\section{Introduction}

Allogeneic and autologous hematopoietic stem cell transplantation (HCT) is considered a routine but complex therapy for patients with otherwise incurable chemo- and immune-sensitive malignant and non-malignant disorders. ${ }^{1}$ The treatment is also used for replacing deficient hematopoietic cells or cellular components and more recently for repairing hematopoietic stem cells by gene editing. Despite its increasing applications and international expansion of access, allogeneic HCT is still associated with significant morbidity and mortality and remains an example of highly specialized, high-cost medicine. It requires extensive experience, significant infrastructure and a network of specialists from all fields of medicine. Over the last two decades in particular, allogeneic HCT has undergone a constant technological evolution, with decreasing transplant related-morbidity and mortality and expansion of the donor pool. This has been achieved by optimizing indications, by manipulating alloreactive immune reactions ex vivo and in vivo and by using novel reduced or minimal intensity conditioning regimens. ${ }^{2}$ As a result, HCT is being offered to patients without a matched donor, to older patients and to those with comorbidities..$^{3-5}$ The predominant autologous HCT transplant type, in contrast, relies exclusively on the highdose preparative regimen for tumor eradication or for reshaping the immune system in autoimmune diseases. ${ }^{6,7}$ Patients' own hematopoietic stem cells are required to rebuild a normal hematopoietic system after the intensive preparative regimen. Missing graft-versus-host disease leads to extremely low mortality, but missing graft-versustumor effects to high relapse rates. It is not surprising that autologous HCT involves different treatment strategies and indications as compared to allogeneic HCT, but like allogeneic HCT it requires extensive experience, significant infrastructure and a network of specialists from all fields of medicine.

However, the increasing specialization and complexity of health care systems required, threaten global equity of access to HCT. The World Health Organization (WHO; www.who.org) declared the transplantation of organs, cells and tissues a global priority and formed a task force to address quality, safety and equity of access. In order to achieve this, analysis of baseline global activity and evolving trends is essential. ${ }^{8}$ The Worldwide Network for Blood and Marrow Transplantation (WBMT; www.wbmt.org), is a non-governmental (NGO) umbrella organization in the field of HCT and in collaboration with the $\mathrm{WHO}$, has taken up the challenge of collecting and disseminating global HCT activity data on a regular basis. Information on indications, the use of different technologies, donor types and trends over time provide a sound basis for physicians to provide appropriate patient counseling and for health care agencies to develop the necessary infrastructure. Informed by global activity survey data, the WBMT performs worldwide workshops to support the development of new HCT programs and to optimize existing programs. The ability to share accumulated experience covering a wide range of strategies, successes and pitfalls continues to be key in improving global HCT access for patients in need.

The first WBMT HCT activity report was based on the global HCT activity in $2006 .^{\circ}$ This was followed by an updated report in 2010. ${ }^{9}$ After reaching the global milestone of one million HCT in $2012,{ }^{10}$ the WBMT focused on analyzing major trends from 2006 onwards ${ }^{11}$ and noted a narrowing of gaps in the African/East Mediterranean (AFR/EMRO) regions. ${ }^{12}$

The success of HCT depends on a number of factors including, the early and effective control of the underlying disease prior to HCT, risk of relapse of the underlying disease and donor characteristics. Over the past decade, the rapid evolution of molecular diagnostic and prognostic techniques has led to the emergence of more accurate prognostic tools and effective targeted molecular therapies for malignant hematological diseases.

We report on the global activity trends between 2014 and 2016 compared to 2006 with a specific focus on global trends in equity of access, in indications of HCT and in donor type. 


\section{Methods}

\section{Study design}

In this retrospective observational survey we analyzed the worldwide HCT activity from the first published series of bone marrow transplants collected from the scientific literature and from member societies for very early transplants. ${ }^{11}$ After 2006, activities were obtained annually through the WBMT network using a unified center-based reporting system. Information from 2016 onwards is given as prediction based on currently available incomplete and non-validated data.

Main outcome measures were the spread of HCT over time and transplant by donor type, country of origin, and WHO region. Secondary outcome measures were to document any trends in the number of HCT by donor type or region, to classify these trends, and quantify differences in the use of autologous or allogeneic HCT across indications and regions.

No individual patient data were used and no ethics committee approval was mandated. Outcome information is not available from our center-specific registry and we opted against the report of fragmented outcome information of just two developed regions (EBMT and CIBMTR).

\section{Data collection and validation}

Global transplant numbers by country of origin, year of transplant, disease and donor type (autologous vs, allogeneic) have been collected since 2006 (foundation year of the WBMT) in 194 WHO member states through the registries of the reporting member organizations, or national registries or transplant centers directly either in paper form or electronically using the standardized WBMT form. Detailed and validated information about main indication including stage of the disease, stem cell source, and allogeneic (family matched, family mismatched and unrelated) donor type were obtained for the years 2006 to 2016. Data were validated by a range of different independent systems; through confirmation by the reporting teams, following receipt of a computer printout of the entered data, by selective comparison with MEDA/TED datasets in the EBMT or CIBMTR data system or by crosschecking for double reporting with national registries. Data were validated by onsite visits to selected teams to verify reported data as part of the quality control program within the European, North American, Latin American and Asia-Pacific organizations. On-site visits to selected teams were part of the quality-control accreditation program of JACIE (www.ebmt.org/jacie-accreditation) or FACT (www.factweb.org). Based on quality controls and contacts with regulatory agencies or national offices, the response rate for allogeneic HCT was estimated to be $>95 \%$ and for autologous HCT 80 $90 \%$. The number of potential missing transplant numbers is estimated to be less than 5\% for allogeneic HCT and less than 15\% for autologous HCT. This number is much lower for Australia, Canada, Europe, Japan, and the USA. The survey focuses on the numbers of patients treated for the first time with HCT.

\section{Participating hematopoietic cell transplantation (HCT) teams, groups, countries and continents}

In 2016, 1,662 HCT teams in 86 countries over six WHO continental regions delivered HCT services globally [(www.who.int/about/regions/en) $)$. These regions included the Americas (AMR/PAHO; WHO regions North-, Middle and South America and Canada); Asia (SEAR/WPR; WHO regions South East Asia and Western Pacific Region, which includes Australia and New Zealand); Europe (EUR; which includes Turkey and Israel) and AFR/EMR (WHO regions Africa and Eastern Mediterranean). For specific analyses AMR/PAHO activities were divided in North America and Latin-America and AFR/EMR in Africa and EMR. A detailed list of organizations providing activity data and definitions used in the manuscript are reported in the Online Supplementary Appendix.

\section{Statistical analysis}

The data analysis was comprised of ordinary least squares regressions for trends, $\chi^{2}$ tests for independent proportions of indications, and binomial tests for donor type. Calculations were done in Eviews ${ }^{8}$ and Excel 2010 (Microsoft).

\section{Results}

\section{Total hematopoietic cell transplantations and overall trends}

From 1957-2016, a total of 1,298,897 HCT (57.1\% autologous) procedures were recorded. The cumulative numbers increased continuously from 10,000 in 1985, to 500,000 in 2005 and doubled to 1 million HCT by 2012. Projecting a frequency of at least 86,844 HCT for 2017 and 89,510 HCT for 2018 (incomplete and not validated data), a total of 1.5 million HCT worldwide was expected to be reached by 2019 (Online Supplementary Figure S1). The annual activity increased continuously from 46,563 in 2006 to 82,718 in 2016, amounting to a global increase of $77.6 \%$ since 2006 , which was somewhat higher in allogeneic $(89.0 \%)$ than in autologous HCT $(68.9 \%$, Table 1$)$. The yearly increase was by a median of $5.9 \%$ for all HCT (allogeneic $6.8 \%$ and autologous $5.9 \%$ ) to a total of 697,934 procedures (54\% autologous) since 2006. The most frequent indications were lymphoproliferative disorders (LPD; $n=370,884$ HCT of which $88.4 \%$ were autologous) and leukemia ( $n=248,860$ total of which $94.9 \%$ were allogeneic; see the Online Supplementary Figure S2). Global HCT team numbers plateaued in the last 4 years with a slight increase in 2016 (Online Supplementary Figure S3), while annual HCT numbers increased continuously. The increase was not a consequence of more reporting HCT teams, but of increased activity per HCT team. While the overall number of HCT per team was 35.1 in 2006, this reached 49.8 in 2016 (Online Supplementary Figure S3). Absolute numbers of all HCT per countries ranged from 0 to 19,505 .

\section{Hematopoietic cell transplantation teams activity in $\mathbf{2 0 1 6}$}

In 2016 , the rates of HCT exceeded 80,000 HCT per year for the first time with 82,718 HCT (53.5\% autologous) reported in the global HCT activity survey (Table 1). The majority of HCT were performed in Europe (45.2\%) and in North America (24.4\%), while SEAR/WPR contributed with $22.7 \%$, Latin-America with $5.1 \%$ and AFR/EMR with $2.7 \%$. The trends for TR were somewhat different, with rates highest in North America (561 TR), followed by Europe (439 TR), Latin America (77 TR), SEAR/WPR (54 TR), EMR (36 TR) and Africa (9 TR) (Figure 1). TR were higher for autologous than for allogeneic HCT in all regions except for SEAR/WPR, EMR and AFR. TR for allogeneic HCT ranged from 0.3 in Morocco to 414.0 in Israel (median 47.6); and for autologous HCT from 0.1 in Egypt to 705.9 in Iceland (median 99.1).

The number of HCT teams varied considerably across regions, with the highest numbers being in SEAR/WPR and Europe (Figure 2). In contrast, TD ranged from 0.05 29.4/country and was highest in Europe (7.7 TD) followed 
Table 1. Global hematopoietic cell transplantation (HCT) activity in 2006, 2016 and changes according to disease indication, donor type and world region.

\begin{tabular}{|c|c|c|c|c|c|c|c|c|c|c|c|c|c|c|c|c|}
\hline & \multicolumn{3}{|c|}{ Family } & \multicolumn{3}{|c|}{ Unrelated } & \multicolumn{3}{|c|}{ Allogeneic } & \multicolumn{3}{|c|}{ Autologous } & \multicolumn{4}{|c|}{ Total } \\
\hline & 2006 & 2016 & $\begin{array}{c}\Delta \% \\
06-16)\end{array}$ & 2006 & 2016 & $\underset{(06-16)}{\Delta \%}$ & 2006 & 2016 & $\underset{(06-16)}{\Delta \%}$ & 2006 & 2016 & $\begin{array}{c}\Delta \% \\
(06-16)\end{array}$ & 2006 & 2016 & $\begin{array}{c}\Delta \% \\
(06-16)\end{array}$ & $\begin{array}{c}2016 \\
\%\end{array}$ \\
\hline Leukemias & 7,491 & 14,992 & 100 & 6,901 & 13,727 & 99 & 14,392 & 28,719 & 99.5 & 1,726 & 844 & -51.1 & 16,118 & 29,563 & 83.4 & 35.7 \\
\hline AML & 3,587 & 7,531 & 110 & 3,024 & 6,803 & 125 & 6,611 & 14,334 & 116.8 & 1,269 & 631 & -50.3 & 7,880 & 14,965 & 89.9 & 18.1 \\
\hline ALL & 1,679 & 3,965 & 136 & 1,809 & 2,930 & 62 & 3,488 & 6,895 & 97.7 & 209 & 155 & -25.8 & 3,697 & 7,050 & 90.7 & 8.5 \\
\hline CML & 826 & 624 & -24 & 508 & 484 & -5 & 1,334 & 1,108 & -16.9 & 14 & 1 & -92.9 & 1,348 & 1,109 & -17.7 & 1.3 \\
\hline MDS/MPS & 1,052 & 2,472 & 135 & 1,216 & 3,144 & 159 & 2,268 & 5,616 & 147.6 & 59 & 10 & -83.1 & 2,327 & 5,626 & 141.8 & 6.8 \\
\hline CLL & 295 & 220 & -25 & 261 & 240 & -8 & 556 & 460 & -17.3 & 173 & 22 & -87.3 & 729 & 482 & -33.9 & 0.6 \\
\hline Other leukemias & 52 & 180 & 246 & 83 & 126 & 52 & 135 & 306 & 126.7 & 2 & 25 & $1,150.0$ & 137 & 331 & 141.6 & 0.4 \\
\hline LPD & 1,863 & 2,023 & 9 & 1,356 & 1,949 & 44 & 3,219 & 3,972 & 23.4 & 21,655 & 39,878 & 84.2 & 24,874 & 43,850 & 76.3 & 53.0 \\
\hline Plasma cell disorders & 490 & 312 & -36 & 283 & 348 & 23 & 773 & 660 & -14.6 & 10,675 & 23,701 & 122.0 & 11,448 & 24,361 & 112.8 & 29.5 \\
\hline HD/NHL & 1,373 & 1,673 & 21 & 1,073 & 1,573 & 47 & 2,446 & 3,246 & 32.7 & 10,980 & 16,103 & 46.7 & 13,426 & 19,349 & 44.1 & 23.4 \\
\hline Lymphoma other/unknown & 0 & 38 & & 0 & 28 & & 0 & 66 & & 0 & 74 & & 0 & 140 & & 0.2 \\
\hline Solid tumors & 110 & 47 & -57 & 40 & 37 & -7 & 150 & 84 & $-44,0$ & 2,560 & 2,853 & 11.4 & 2,710 & 2,937 & 8.4 & 3.6 \\
\hline Non-malignant disorders & 1,458 & 3,423 & 134 & 902 & 2,004 & 122 & 2,360 & 5,427 & 139.4 & 193 & 691 & 258.0 & 2,553 & 6,118 & 139.6 & 7.3 \\
\hline Bone marrow failures & 840 & 1,775 & 111 & 452 & 909 & 101 & 1,292 & 2,684 & 107.7 & 0 & 6 & & 1,292 & 2,690 & 108.2 & 3.3 \\
\hline Hemoglobinopathies & 338 & 962 & 185 & 54 & 301 & 457 & 392 & 1,263 & 222.2 & 3 & 10 & 233.3 & 395 & 1,273 & 222.3 & 1.5 \\
\hline Immune deficiencies & 206 & 472 & 129 & 239 & 500 & 109 & 445 & 972 & 118.4 & 3 & 15 & 400.0 & 448 & 987 & 120.3 & 1.2 \\
\hline $\begin{array}{l}\text { Inherited diseases } \\
\text { of metabolism }\end{array}$ & 59 & 111 & 88 & 118 & 182 & 54 & 177 & 293 & 65.5 & 2 & 4 & 100.0 & 179 & 297 & 65.9 & 0.4 \\
\hline Autoimmune disorders & 6 & 19 & 217 & 10 & 22 & 120 & 16 & 41 & 156.3 & 184 & 637 & 246.2 & 200 & 678 & 239.0 & 0.8 \\
\hline $\begin{array}{l}\text { Other non-malignant } \\
\text { disorders }\end{array}$ & 9 & 84 & 833 & 29 & 90 & 210 & 38 & 174 & 357.9 & 1 & 19 & $1,800.0$ & 39 & 193 & 394.9 & 0.2 \\
\hline Others & 80 & 109 & 36 & 132 & 114 & -14 & 212 & 223 & 5.2 & 96 & 27 & -71.9 & 308 & 250 & -18.8 & 0.3 \\
\hline EUR & 4,906 & 7,074 & 44 & 4,222 & 8,348 & 98 & 9,128 & 15,422 & 69.0 & 15,088 & 21,946 & 45.5 & 24,216 & 37,368 & 54.3 & 45.2 \\
\hline North America & 2,580 & 3,680 & 42.6 & 2,878 & 4,458 & 54.9 & 5,458 & 8,138 & 49.1 & 6,730 & 12,006 & 78.4 & 12,188 & 20,144 & 65.3 & 24.4 \\
\hline SEARWPR & 1,948 & 7,392 & 279 & 2,110 & 4,513 & 114 & 4,058 & 11,905 & 193.4 & 3,038 & 6,884 & 126.6 & 7,096 & 18,789 & 164.8 & 22.7 \\
\hline Latin America & 771 & 1,197 & 55.3 & 115 & 438 & 280.9 & 886 & 1,635 & 84.5 & 947 & 2,561 & 170.4 & 1,833 & 4,196 & 128.9 & 5.1 \\
\hline EMR/AFR & 797 & 1,251 & 57 & 6 & 74 & 1,133 & 803 & 1,325 & 65.0 & 427 & 896 & 109.8 & 1,230 & 2,221 & 80.6 & 2.7 \\
\hline TOTAL & 11,002 & 20,594 & 87.0 & 9,331 & 17,831 & 91.0 & 20,333 & 38,425 & 89.0 & 26,230 & 44,293 & 68.9 & 46,563 & 82,718 & 77.6 & 100 \\
\hline
\end{tabular}

$\Delta(06 / 16)$ Difference from 2006 to 2016 in \%; AML: acute myeloid leukemia; ALL: acute lymphoblastic leukemia; CML: chronic myeloid leukemia; MDS/MPS: myelodysplastic syndrome/myeloproliferative syndrome; CLL: chronic lymphocytic leukemia; LPD: lymphoproliferative disorders; EUR: Europe; SEAR/WPR: South East Asia Pacific Region/West Pacific Region; EMR/AFR: East Mediterranean Region/African Region.

by North America (6.0 TD), SEAR/WPR (1.9 TD), Latin America (1.6 TD), EMR (0.4 TD) and Africa (0.3 TD). Accordingly, the most HCT per team were performed in North America and EMR.

\section{Trends in indications and hematopoietic cell transplantation type}

All regions showed increases in activity compared to 2006 (range, 54.3\% in EUR - 164.8\% in SEAR/WPR) for both transplant types (range, autologous $45.5 \%$ in EUR $126.6 \%$ in SEAR/WPR and allogeneic 49.1\% North America - 193.4\% SEAR/WPR; Table 1). Trends and increase in allogeneic and autologous HCT from 2006 to 2016 according to disease and regions are shown in Figure 3 and the Online Supplementary Figure S4A and B, respectively. The most common indications for autologous and allogeneic HCT $(n=82,718)$ were lymphoproliferative diseases $(53.0 \%)$, leukemias $(35.7 \%)$, non-malignant disorders $(7.3 \%)$ and solid tumors $(3.6 \%$; see Table 1$)$.

\section{Allogeneic hematopoietic cell transplantations}

Leukemia is the most common indication for allogeneic HCT $(n=28,719)$ and the most frequent single indication acute myeloid leukaemia (AML) with a total of 14.334 HCT, followed by acute lymphoblastic leukemia (ALL; $\mathrm{n}=6895$ ), myelodysplastic syndrome (MDS)/myeloproliferative neoplasms (MPS; $\mathrm{n}=5,616$ ) and chronic myeloid leukemia (CML; $n=1,108$ ). Non-malignant disorders $(\mathrm{n}=5,427)$ with the subgroups bone marrow (BM) failure $(49.5 \%)$, hemoglobinopathies $(23.3 \%)$ and immunodeficiencies $(17.9 \%)$ were identified as the second most common indication for allogeneic HCT. The third most common indication was lymphoproliferative disorder (LPD; $\mathrm{n}=3,972$ ) of which $81.7 \%$ were Hodgkin disease (HD)/non-Hodgkin disease (NHL) and $16.6 \%$ plasma cell disorders (PCD).

Increases from 2006 were noted especially in non-malignant disorders (139.4\%), leukemias $(99.5 \%)$ with the subgroups MDS/MPS (147.6\%), AML (116.8\%) and LPD $(23.4 \%)$. Declines were noted in CML (-16.9\%) and chronic lymphocytic leukemia (CLL) (-17.3\%; Table 1). HCT were performed predominantly in AML in first complete remission (CR1) (Online Supplementary Figure S4A) with a decline in procedures in non-CR1. In ALL CR1 was also more frequent than non-CR1. HCT for MDS and, to a lower extent MPN, increased steadily over the observa- 


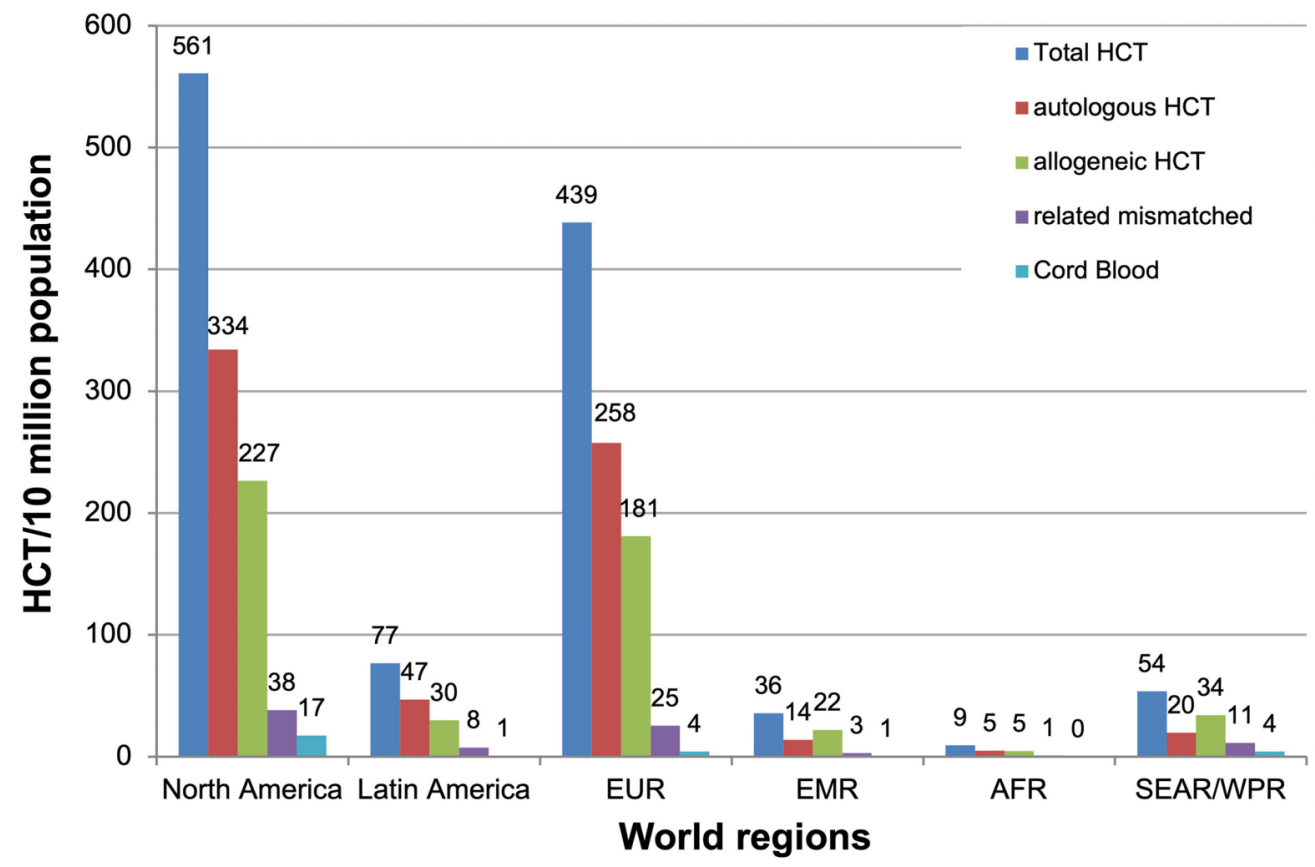

Figure 1. Transplant rates. Hematopoietic cell transplantation (HCT)/10 million population according to transplant type (autologous, allogeneic, related mismatched and cord blood) and world regions in 2016. EUR: Europe, EMR: East Mediterranean Region; AFR: Africa; SEAR/WPR: South East Asia Pacific Region/West Pacific Region.

tion period. Increased allogeneic HCT activities were observed for almost all indications in all regions. The highest increase was noted for related donors in the SEAR/WPR region (279\%; Table 1; Figure 3). The only observed decrease involved solid tumors (44\% decrease) in almost all regions except for SEAR/WPR and LPD in North America.

\section{Autologous hematopoietic cell transplantation}

The most frequent indication for autologous HCT in 2016 was LPD ( $n=39,878,84.2 \%$ of all autologous HCT; Table 1) and the most frequent single indication PCD and $\mathrm{HD} / \mathrm{NHL}$ with $59.4 \%$ and $40.4 \%$, respectively. HCT for solid tumors $(n=2,853)$ accounted for $6.4 \%$ of autologous HCT and $844(1.9 \%)$ were performed for leukemias mostly for AML $(74.8 \%)$ and ALL $(18.4 \%)$. Autoimmune diseases (AID) $(92.2 \%)$ were the predominant indication for autologous HCT within non-malignant diseases $(n=691)$. Frequencies of autologous HCT increased in almost all indications and regions especially for LPD and non-malignant disorders. Decreases in autologous HCT were observed for leukemia (except SEAR/WPR) and for solid tumors in Europe. The highest increase in autologous HCT was observed in Latin America for non-malignant disorders. The frequency of autologous HCT increased by $68.9 \%$ predominantly in non-malignant disorders (258\%), primarily AID $(246.2 \%)$ in PCD $(122 \%)$ and in lymphoma (46.7\%; Online Supplementary Figure 4B; Table 1). Decreased activity of autologous HCT was reported for all leukemias (-51.1\%; Figure 3).

\section{Trends in donor type and stem cell source}

Autologous HCT (range, 0-11,655) were reported from 85 participating countries, while allogeneic HCT (range, 0$7,850)$ were reported from 76 countries, including procedures from unrelated donors and from CB in 55 and in 41 countries, respectively. Overall, related HCT has become more frequent than unrelated HCT starting in 2014 (Online Supplementary Figure S5). The increase in related HCT was mainly due to the use of non-identical related donors $(39.5 \%$ of related HCT), which increased significantly over the last 4 years, while unrelated $\mathrm{CB}$ showed a moderate decline. A detailed analysis of identical and nonidentical related HCT according to indication and in comparison to unrelated HCT is given in Table 2. Highest increases were observed for severe aplastic anemia and hemoglobinopathies (Delta 2007-2016 >1,000\%) and for leukemia (Delta 2007-2016 >550\%; especially AML CR1, ALL CR1, CML first CP with maximum 2,456\%, data not shown), but also in LPD (especially in HD and NHL max Delta $2007-2016=711 \%$ ). In comparison, HCT from related identical donors showed Delta 2007-2016 of maximum $189 \%$ for ALL CR1 and from unrelated for AML CR1 of $430 \%$.

Amongst allogeneic HCT, the proportion of unrelated donor HCT ranged from $4.4 \%$ to $78.3 \%$ (median $20 \%$ ), with 30 countries performing more unrelated than related donor HCT. Absolute unrelated HCT numbers ranged from 0 to 4,311 and those of $C B$ ranged from 0 to 1,233 in individual countries. It is not surprising that more related HCT were performed in regions without an unrelated donor registry (Latin America, EMR, AFR and SEAR/WPR). Related haploidentical HCT $(n=8,131)$ were evenly distributed in 62 countries; with absolute numbers ranging from 0 to 2,554 and proportions of all allogeneic HCT ranging between $1.5 \%$ to $77 \%$ (median $8 \%$ ). TR for haploidentical HCT was highest in North America $(n=38)$, 25 in Europe, eight in Latin America and $\leq 4$ in Asia Pacific, AFR and EMR (Figure 1). However, TR as \% of allogeneic HCT reached $34 \%$ in SEAR/WPR and $26 \%$ in Latin America, while it was only $14 \%$ in Europe and EMR.

Peripheral blood (PB) was the predominant graft source in both autologous (99.7\%) and allogeneic $(72.8 \%)$ HCT, while CB as a source has declined (in 2016 13.9\% of all 
Table 2. Trend of global hematopoietic cell transplantation activity according to disease indication and donor type.

\begin{tabular}{|c|c|c|c|c|c|c|c|c|c|c|c|c|}
\hline & \multicolumn{3}{|c|}{$\begin{array}{l}\text { Related } \\
\text { identical }\end{array}$} & \multicolumn{3}{|c|}{$\begin{array}{c}\text { Related } \\
\text { non-identical }\end{array}$} & \multicolumn{3}{|c|}{ Related total } & \multicolumn{3}{|c|}{ Unrelated } \\
\hline & 2007 & 2016 & $\Delta \%(07-16)$ & 2007 & 2016 & $\Delta \%(07-16)$ & 2007 & 2016 & $\Delta \%(07-16)$ & 2007 & 2016 & $\Delta \%(07-16)$ \\
\hline Leukemias & 6,858 & 8,906 & 29.9 & 1,101 & 6,056 & 450.1 & 8,018 & 14,992 & 87.0 & 7,868 & 13,727 & 74.5 \\
\hline AML & 3,249 & 4,453 & 37.1 & 472 & 3,064 & 549.2 & 3,749 & 7,531 & 100.9 & 3,610 & 6,803 & 88.5 \\
\hline ALL & 1,635 & 2,256 & 38.0 & 376 & 1,702 & 352.7 & 2,023 & 3,965 & 96.0 & 2,015 & 2,930 & 45.4 \\
\hline CML & 601 & 388 & -35.4 & 56 & 236 & 321.4 & 663 & 624 & -5.9 & 479 & 484 & 1.0 \\
\hline MDS/MD/MPN & 1,044 & 1,559 & 49.3 & 156 & 905 & 480.1 & 1,211 & 2,472 & 104.1 & 1,358 & 3,144 & 131.5 \\
\hline CLL & 279 & 166 & -40.5 & 17 & 53 & 211.8 & 298 & 220 & -26.2 & 316 & 240 & -24.1 \\
\hline Other leukemias & 50 & 84 & 68.0 & 24 & 96 & 300.0 & 74 & 180 & 143.2 & 90 & 126 & 40.0 \\
\hline LPD & 1,677 & 1,238 & -26.2 & 163 & 758 & 365.0 & 1,877 & 2,023 & 7.8 & 1,338 & 1,949 & 45.7 \\
\hline Plasma cell disorders & 542 & 234 & -56.8 & 23 & 64 & 178.3 & 583 & 312 & -46.5 & 245 & 348 & 42.0 \\
\hline Multiple myelomas & 341 & 211 & -38.1 & 8 & 54 & 575.0 & 356 & 279 & -21.6 & 192 & 316 & 64.6 \\
\hline Others & 9 & 23 & 155.6 & 1 & 10 & 900.0 & 11 & 33 & 200.0 & 7 & 32 & 357.1 \\
\hline Lymphoma & 1,135 & 979 & -13.7 & 140 & 681 & 386.4 & 1,294 & 1,673 & 29.3 & 1,093 & 1,573 & 43.9 \\
\hline Hodgkin disease & 186 & 205 & 10.2 & 29 & 170 & 486.2 & 222 & 377 & 69.8 & 175 & 181 & 3.4 \\
\hline Non-Hodgkin lymphoma & 796 & 774 & -2.8 & 63 & 511 & 711.1 & 871 & 1,296 & 48.8 & 683 & 1,392 & 103.8 \\
\hline Solid tumors & 47 & 4 & -91.5 & 32 & 42 & 31.3 & 84 & 47 & -44.1 & 35 & 37 & 5.7 \\
\hline Non-malignant disorders & 1,354 & 2,185 & 61.4 & 196 & 1,232 & 528.57 & 1,559 & 3,423 & 119.56 & 984 & 2,004 & 103.66 \\
\hline Bone marrow failure & 789 & 1,136 & 44.0 & 65 & 633 & 873.9 & 862 & 1,775 & 105.9 & 448 & 909 & 102.9 \\
\hline Severe aplastic anemia & 644 & 964 & 49.7 & 48 & 553 & $1,052.1$ & 700 & 1,523 & 117.6 & 320 & 743 & 132.2 \\
\hline Other & 145 & 172 & 18.6 & 17 & 80 & 370.6 & 162 & 252 & 55.6 & 128 & 166 & 29.7 \\
\hline Hemoglobinopathies & 338 & 711 & 110.4 & 21 & 251 & $1,095.2$ & 359 & 962 & 168.0 & 66 & 301 & 356.1 \\
\hline Immune deficiencies & 140 & 234 & 67.1 & 84 & 238 & 183.3 & 224 & 472 & 110.7 & 255 & 500 & 96.1 \\
\hline Inherited diseases of metabolism & 44 & 54 & 22.7 & 19 & 57 & 200.0 & 63 & 111 & 76.2 & 98 & 182 & 85.7 \\
\hline Autoimmune disorders & 12 & 10 & -16.7 & 1 & 9 & 800.0 & 13 & 19 & 46.2 & 8 & 22 & 175.0 \\
\hline Other non malignant disorders & 31 & 40 & 29.0 & 6 & 44 & 633.3 & 38 & 84 & 121.1 & 109 & 90 & -17.4 \\
\hline Other & 61 & 66 & 8.2 & 12 & 43 & 258.3 & 73 & 109 & 49.3 & 68 & 114 & 67.7 \\
\hline TOTAL & 9,997 & 12,399 & 24.0 & 1,504 & 8,131 & 440.6 & 11,611 & 20,594 & 77.4 & 10,293 & 17,831 & 73.2 \\
\hline
\end{tabular}

unrelated and $6.7 \%$ of all allogeneic HCT). Of the 38,425 allogeneic HCT, 7,868 (20\%) were bone marrow (BM)derived, 27,963 (73\%) were from $\mathrm{PB}$ and 2,594 (7\%) from cord blood (CB). Of the 44,293 autologous HCT, $99.7 \%$ were from $\mathrm{PB}, 0.3 \%$ from $\mathrm{BM}$ and only three single $\mathrm{HCT}$ were from $\mathrm{CB}$.

\section{Discussion}

The analysis of global HCT activity based on data from 2006-2016 on 700.000 HCT over the last 10 years gathered by the WBMT gives important insights into the global trends in the field of HCT.

As per previous reports, ${ }^{8-13}$ HCT activities continue to increase worldwide without plateau and exceeded 80,000 procedures annually for the first time in 2016 and are expected to exceed 90,000 in 2018 to reach a total of 1.5 million by 2019. Although allogeneic HCT has increased more than autologous in recent years, the later remains the predominant transplant type and LPD the leading disease indication. Leukemia is the second most frequent HCT indication and $94.9 \%$ of these use allogeneic donors. While HCT on patients with acute leukemias (predominantly in CR1, but not in non-CR1) and MDS/MPN increase, a decrease of frequencies in chronic leukemias (CLL and CML) was observed. Non-malignant disorders now account for $7.3 \%$ of all HCT (89.2\% of which are allogeneic) with BM failures as the most frequent group of disease indications $(47.5 \%$, mostly severe aplastic anemia). Hemoglobinopathies were the second most frequent indication (22.4\%) with an increase of $222.3 \%$. Finally, solid tumors were the indication in $3.6 \%$ of all HCT and were almost exclusively transplanted with autologous grafts (97\%). Striking is the pronounced increase in haploidentical HCT in hemoglobinopathies, severe aplastic anemia, leukemia and LPD (especially MM and NHL) in developing countries. Findings are partly in agreement on a larger scale with the development in Europe and US, but also diverge especially on the use of haploidentical HCT for non-malignant diseases, in the use of autologous HCT in autoimmune disease (LABMT) and in leukemias (SEAR/WPR) and in the use of allogeneic HCT in autoimmune diseases in SEAR/WPR.

There was substantial divergence in the rate of growth of HCT between different regions, with increases of more than $129 \%$ in SEAR/WPR and Latin-America over the last decade contributing strongly to the continuous global upward trend in activity. Both regions reported their major increases in non-malignant disorders, in the SEAR/WPR with allogeneic and in Latin America with autologous HCT. AFR/EMR had a remarkable growth of $80 \%$ in comparison to $65 \%$ in North America and 54\% in Europe. Despite the differential increase, TR still vary by more than 10 -fold from region to region. A deeper analysis of the number of HCT teams in 


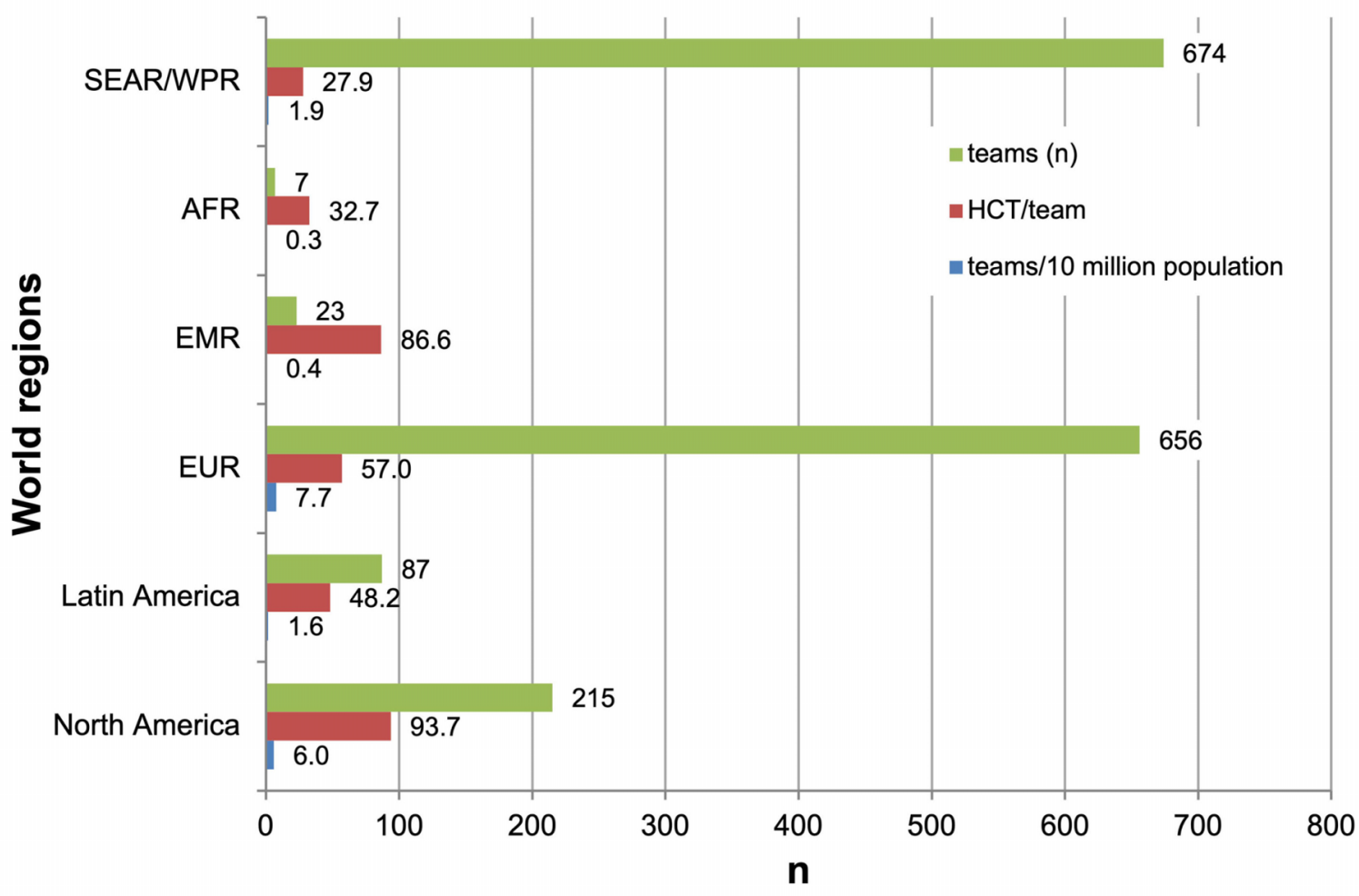

Figure 2. Number of hematopoietic cell transplant (HCT) teams, HCT team density and HCT per team according to world regions. EUR: Europe; EMR: East Mediterranean Region; AFR: Africa; SEAR/WPR: South East Asia Region/Western Pacific Region; TD: team density.

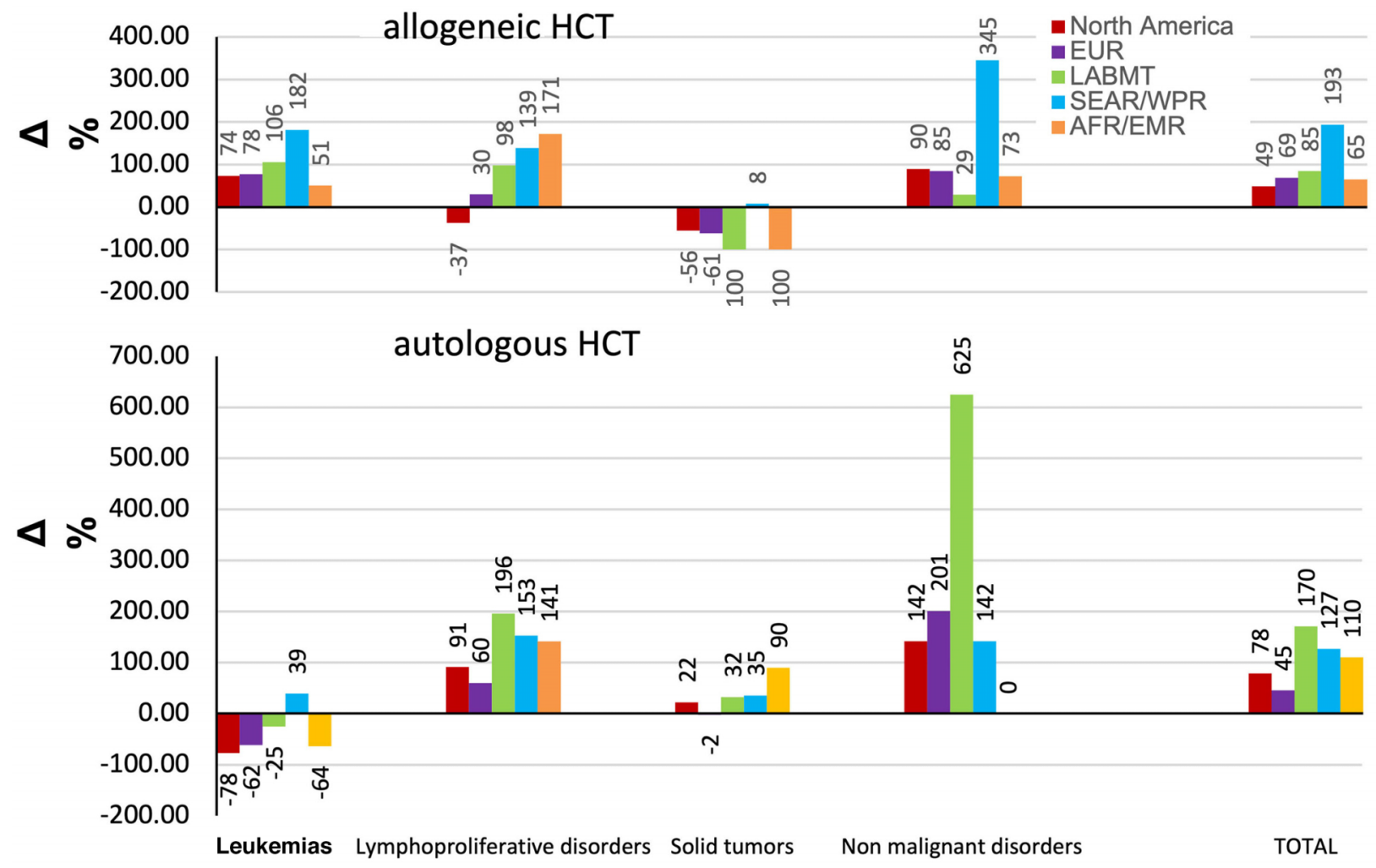

Figure 3. Change in activity in percent from 2006 and 2016 according to disease indication and world region. HCT: hematopoietic cell transplantation; EUR: Europe; LABM: Latin American Blood and Marrow Transplantation Group; TAFR/EMR: Africa/East Mediterranean Region;; SEAR/WPR: South East Asia Region/Western Pacific Region. 
the different regions showed three patterns: high $(>500$ teams; SEAR/WPR and EUR), intermediate (100-500 teams; North America) and low (<100 teams; AFR, EMR, Latin America). TD was similar in North America and Europe (6.0 and 7.7 respectively), between 1-2 in SEAR/WPR and Latin America and $<1$ in all other regions. While North America and EMR had most HCT per HCT team (93.7 and 86.6, respectively), Europe and Latin America had intermediate (57.0 and 48.2, respectively), SEAR/WPR and AFR had the lowest (27.9 and 32.7, respectively) HCT per team. It is encouraging that HCT and TR in developing regions are increasing steadily, although there are differences in indications and diseases, and are closing the HCT access gap. However, the disparities in access between regions remains substantial.

Although the WBMT has established a global coverage of international transplant societies with an estimated $>90 \%$ reporting, at least one very large country had incomplete reporting and lacked a national registry. With the aim of establishing such a registry, the WBMT organized a successful workshop to highlight the importance of coordinated reporting. Increased numbers of teams and activities in that country are now expected starting in 2016 (Xu L-P, Lu P_H, Wu D-P et al. Hematopoietic stem cell transplantation activity in China 2019: a report from the Chinese Blood and Marrow Transplantation Registry Group. Bone marrow transplantation; 2021 [in press]). Therefore, the data presented here are certainly an underestimate but are expected to increase further with greater completeness of reporting. ${ }^{14}$

The considerable increase seen in the SEAR/WPR and Latin American region in non-malignant disorders, hemoglobinopathies (allogeneic HCT) and AID (autologous HCT, predominantly in multiple sclerosis), is of special interest. It is reassuring to see a worldwide trend of more patients to be transplanted in CR1 rather than later in their disease course. This phenomenon is much more evident in AML and remains constant in ALL. Lymphoma accounts for less of the increase in autologous HCT than do PCD. The indications for CML decreased only slightly in the 10 year period following the availability of successive tyrosine kinase inhibitor pharmaceuticals. However, it should be noted here that more patients were transplanted beyond chronic phase CML, and not in line with ELN recommendations. ${ }^{15}$ The changes in donor type and graft source confirm trends previously described. Frequencies of haploidentical HCT increased considerably to reach $>10 \%$ of all allogeneic HCT throughout all regions. As a consequence, more related (matched and haploidentical) HCT were performed than were unrelated HCT, and the later appears to have reached a plateau. The increase of haploidentical related donors might have several reasons including lack of donors for Latin-American patients in international registries, lack of local national donor registries and economic. The availability of new technologies like the postcyclophosphamid protocol and the so called 'Beijing Protocol' using G-CSF/anti-thymocyte globulin and multiple stem cell sources (BM and PB stem cells) ${ }^{14,16}$ may have contributed to this development. Overall, CB HCT decreased consistent with the pattern seen in Europe.

There are a few limitations in this analysis. The first one relates to the reporting lag of the analysis. Logistics in obtaining data from 1,660 transplant centers worldwide are the main cause for this delay. While some of the regions are reporting in real time, emerging regions have not a central national or regional reporting system. In these regions, WBMT collected the information from individual transplant centers returning the results to the regional societies for completeness checking. An internet-based reporting system was developed by the WBMT and is expected to gradually bring up the survey reports to real time. An additional limitation of this analysis is the inability to provide information on utilization of HCT for specific illnesses and on information restricted to first HCT. Utilization is currently being analyzed worldwide for myeloma and AML by the WBMT, but should be extended to major indications. Furthermore, information on second or third HCT is not currently available, although these account for approximately $10 \%$ of HCT in developed countries, but will be implemented with the new reporting system.

One of the challenges that the WBMT faces, is how to go beyond calculations of global HCT activity and accelerate global equity of access to HCT. The most efficient method may be to increase HCT activity/team. In fact, transplant teams have already increased their annual HCT/team by a median of $14 \mathrm{HCT} /$ team since 2006. As shown in this study, $50 \mathrm{HCT} /$ team are feasible even in developing countries and almost $100 \mathrm{HCT} /$ team are currently being performed in North America.

Increasing team numbers might be more difficult. This is due in part to the funds allocated to local health expenditure, but also due to limitations in the infrastructure required including blood banks, intensive care units, multidisciplinary teams and microbiology expertise. Shortages and unavailability of medicines and lack of trained biomechanical/biotechnical technicians have also hindered HCT activities in developing countries. The WBMT has the capacity to review and analyze global HCT activity data and apply this data to support HCT activities globally. Global activity survey data has been instrumental in informing and shaping HCT support workshops in the different regions, which have been successful in fostering collaboration amongst international societies and supportive expert HCT networks globally. The WBMT, on its site, has already prepared a variety of documents to facilitate the establishment of new transplant programs (requirements for establishing a program, list of essential medicines, use of biosimilars to reduce costs and establishing unrelated donor registries). ${ }^{17-21}$ In addition, supervisory telemedicine is an evolving and potentially powerful tool to overcome lack of experience with collateral benefits for conventional hematology, blood banking, microbiology and virology. Devoted physicians and willing health authorities are essential for the application of such technologies and successful collaborations accompanied by demonstration of compliance with international standards to provide reassurance to internal and external stakeholders. $^{22}$

The achievements obtained in the last decade should be an incentive to continue and even increase the common efforts to improve access and close the gap worldwide faster. This is a common effort of professional organizations, $\mathrm{WHO}$, politicians and Health Authorities. The role of the WHO is essential in coordinating this process among their member states.

\section{Disclosures}

No conflicts of interest to disclose.

\section{Contributions}

$D N, H B, M A, K B, K F$, and FRA designed the study; $H B$, $N B, C B, N C, S C, A E, C F, S G, N H, A A H, S H, A H, M H$, $M I, G J, A K, J K, N K, R P L, J W L, J M R, M P, J P, K P, A S, J A S$, $A S, J S, D W, N W, M K, M A, H G, Y A, W S$ contributed data 
and assured quality of the data given to the analysis; $H B, D N$, $M P$, and LF analysed data; DN, HB, WS, and NH drafted the manuscript; $D N, H B, N B, C B, N C, S C, A E, C F, S G$, $N H, A A H, S H, A H, M H, M I, G J, A K, J K, N K, R P L, J W L$, $J M R, M P, J P, K P, A S, J A S, A S, J S, D W, N W, M K, M A, H G$, $Y A$ processed the manuscript. European data were derived from the European Society for Blood and Marrow Transplantation (EBMT) database for the years 1965-89 and from the EBMT annual activity survey office since 1990. Noneuropean data were initially provided by the Center for International Blood and Marrow Transplant Research (CIBMTR) since 1964. They were supplemented or replaced by the activity surveys of the Asian Pacific Blood and Marrow Transplantation Group (APBMT) since 1974, the Australasian Bone Marrow Transplant Recipient Registry (ABMTRR) since 1992, the Eastern Mediterranean Blood and Marrow Transplantation Group (EMBMT) since 1984, the Cell Therapy Transplant Canada (CTTC) since 2002, the Latin American Bone Marrow Transplantation Group (LABMT) since 2009, and the African Blood and Marrow Transplant Group (AFBMT) since 2010. Unrelated donor and cord blood information were derived from the World Marrow Donor Association (WMDA) and Bone Marrow Donors Worldwide (BMDW).

\section{Acknowledgments}

The cooperation of all participating teams, countries and organizations with their staff is greatly appreciated, specifically the following: ABMTRR: St. Vincent's Hospital Sydney, APBMT: Aichi Medical University, CTTC: Centre Tecnològic de Telecomunicacions de Catalunya; CIBMTR: Medical College of Wisconsin, EBMT: Coordination Offices in Barcelona, Paris and London and the Austrian Registry (ASCTR), the Czech BMT Registry, the French Registry (SFGM), the German Registry (DRST), the Italian Registry (GITMO), the Dutch Registry (HOVON), the Spanish BMT Registry (GETH), the Swiss Registry (SBST), the Turkish BMT Registry and the British Registry (BSBMT), EMBMT, SBTMO, LABMT, AFBMT, WMDA, and Eurocord. The authors thank Michael Cross for editorial work on the manuscript.

\section{Funding}

Funding for this study was indirectly provided by support of the WBMT. Funding was solely to support the study; no individual payment was made to any of the persons involved in the study. The Activity Survey Office is partly supported by the University Hospital of Basel. MG was supported by SCCER CREST. The Online Supplementary Appendix shows details about funding of the participating institutions.

\section{References}

1. Thomas ED, Lochte HL, Lu WC, Ferrebee $\mathrm{JW}$. Intravenous infusion of bone marrow in patients receiving radiation and chemotherapy. New Engl J Med. 1957 (257):491-496.

2. Gratwohl A, Hermans J, Goldman J, et al. Risk assessment for patients with chronic myeloid leukaemia before allogeneic blood or marrow transplantation. Lancet. 1998; 352(9134):1087-1092

3. McSweeney PA, Niederwieser D, Shizuru JA, et al. Hematopoietic cell transplantation in older patients with hematologic malignancies: replacing high-dose cytotoxic therapy with graft-versus-tumor effects. Blood. 2001;97(11):3390-3400.

4. Bacigalupo A. Second EBMT Workshop on reduced intensity allogeneic hemopoietic stem cell transplants (RI-HSCT). Bone Marrow Transplant. 2002;29(3):191-195.

5. Giralt S, Ballen K, Rizzo D, et al. Reducedintensity conditioning regimen workshop: defining the dose spectrum. Report of a workshop convened by the center for international blood and marrow transplant research. Biol Blood Marrow Transplant. 2009;15(3):367-369.

6. Attal G, Harousseau JL, Stoppa AM, et al. A prospective, randomized trial of autologous bone marrow transplantation and chemotherapy in multiple myeloma. $\mathrm{N}$ Engl J Med. 1996;335(2):91-97.

7. Snowden JA, Badoglio M, Labopin M, et al. Evolution, trends, outcomes, and economics of hematopoietic stem cell transplantation in severe autoimmune diseases 4. Blood Adv. 2017;1(27):2742-2755

8. Gratwohl A, Baldomero H, Aljurf M, et al. Hematopoietic stem cell transplantation: a global perspective. JAMA. 2010; 303(16): 1617-1624.

9. Gratwohl A, Baldomero H, Gratwohl M, et al. Quantitative and qualitative differences in use and trends of hematopoietic stem cell transplantation: a Global Observational Study. Haematologica. 2013;98(8):1282-290.

10. Gratwohl A, Pasquini MC, Aljurf M, et al. One million haemopoietic stem-cell transplants: a retrospective observational study. Lancet Haematol. 2015;2(3):e91-100.

11. Niederwieser D, Baldomero $H$, Szer J, Gratwohl M, Aljurf M, Atsuta Y, et al. Hematopoietic stem cell transplantation activity worldwide in 2012 and a SWOT analysis of the Worldwide Network for Blood and Marrow Transplantation Group including the global survey. Bone Marrow Transplant. 2016;51(6):778-785.

12. Baldomero $\mathrm{H}$, Aljurf $M$, Zaidi SZA, et al. Narrowing the gap for hematopoietic stem cell transplantation in the EastMediterranean/African region: comparison with global HSCT indications and trends. Bone Marrow Transplant. 2019;54(3):402417.

13. Iida M, Dodds A, Akter MR, et al. The 2016 APBMT Activity Survey Report: trends in haploidentical and cord blood transplantation in the Asia-Pacific region. Blood Cell Therapy. 2021(4):20-28.

14. Niederwieser D. The Chinese HCT survey: a non-manipulated haploidentical transplantation procedure makes a novel contribution to data sharing within the regional and global transplant registries and to worldwide knowledge. Bone Marrow Transplant. 2021:56(6):1229-1231.

15. Hochhaus A, Baccarani M, Silver RT, et al. European LeukemiaNet 2020 recommendations for treating chronic myeloid leukemia. Leukemia. 2020;34(4):966-984.

16. Xu L-P, Wu D-P, Han M-Z, et al. A review of hematopoietic cell transplantation in China: data and trends during 2008-2016 [eng]. Bone Marrow Transplant. 2017; 52(11): 1512-1518.
17. Fakih RE, Greinix $H$, Koh $M$, et al. Worldwide Network for Blood and Marrow Transplantation (WBMT) recommendations regarding essential medications required to establish an early stage hematopoietic cell transplantation program. Transplant Cell Ther. 2021;27(3):267.e1-267.e5.

18. Muhsen IN, Hashmi SK, Niederwieser D, et al. Worldwide Network for Blood and Marrow Transplantation (WBMT) perspective: the role of biosimilars in hematopoietic cell transplant: current opportunities and challenges in low- and lower-middle income countries. Bone Marrow Transplant. 2020:55(4):698-707.

19. Pasquini MC, Srivastava A, Ahmed SO, et al Worldwide Network for Blood and Marrow Transplantation (WBMT) recommendations for establishing a hematopoietic cell transplantation program (part I): minimum requirements and beyond. Hematol Oncol Stem Cell Ther. 2020;13(3):131-142

20. Aljurf M, Weisdorf D, Hashmi SK, et al Worldwide Network for Blood and Marrow Transplantation (WBMT) recommendations for establishing a hematopoietic stem cell transplantation program in countries with limited resources (part II): clinical, technical and socio-economic considerations. Hematol Oncol Stem Cell Ther. 2020;13(1):7-16.

21. Aljurf M, Weisdorf D, Alfraih F, et al. "Worldwide Network for Blood \& Marrow Transplantation (WBMT) special article, challenges facing emerging alternate donor registries". Bone Marrow Transplantat. 2019;54(8):1179-1188.

22. Frutos C, Enciso ME, Glasenapp A von, Quiroz A, Batista J, Niederwieser D. Bridging the gap using telemedicine: optimizing an existing autologous hematopoietic SCT unit into an allogeneic hematopoietic SCT unit in Paraguay with the help of the WBMT. Blood Adv. 2019;3(Suppl 1):S45-7. 